\title{
COMPARISON OF CONTACT STRESS DISTRIBUTION FOR FOAM SEAT AND SEAT OF AUXETIC SPRING SKELETON
}

\author{
M. JANUS-MICHALSKA* and D. JASIŃSKA \\ Institute of Structural Mechanics \\ Cracow University of Technology \\ ul. Warszawska 24, 31-155 Kraków, POLAND \\ E-mail:mjm@limba.wil.pk.edu.pl \\ jasinska@limba.wil.pk.edu.pl \\ J. SMARDZEWSKI \\ Department of Furniture Design \\ Poznań University of Life Science \\ ul. Wojska Polskiego 28, 60-637 Poznań, POLAND \\ E-mail: jsmardzewski@up.poznan.pl
}

\begin{abstract}
The objective of this paper is to present and compare the results of numerical solutions of contact problem for two types of seats subjected to typical sitting loadings. The first seat is made of a typical hyperelastic foam, the other is designed with an auxetic polyamid spring skeleton. Computer simulations of the seat structure under a typical static loading exerted by a human body are performed by means of ABAQUS FEA. The model provides an insight into deformation modes and stress field in relation to geometric and material parameters of the seat structure. The other type of seat, due to the fact of global auxecity and progressive springs characteristics reduces contact stress concentrations, giving an advantegous distribution of pressure and provides the sensation of physical comfort. The proper seat skeleton shape leads to an improvement of ergonomic quality.
\end{abstract}

Key words: auxetic structures, seat, utility load, contact problem.

\section{Introduction}

The contact interaction between a human body and a seat is the main factor of feeling comfort when seated. In the first stages of the design process a seat should be tested for its degree of comfort by computer simulations with models of the human body and the seat. Seating comfort is becoming increasingly important, due to the fact that people spend more and more time in the sitting position. Many research studies indicate that comfort is primarily associated with the physiological and biomechanical factors. Ebe and Griffin (2001), Zhao et al. (1994), Park and Kim (1997) report a dependence of subjective personal sensation of comfort and average pressure, maximum pressure, the size and symmetry of the contact area. A correct pressure distribution is crucial to seated comfort. Surface pressure is responsible for constriction of blood vessels in underlying tissues, restricting blood flow, which the sitter experiences as discomfort. Because of the large variance in peak pressure patterns among people of different sizes and shapes, it is difficult to design ideal seat and back contours or cushion compliance levels that would minimize uncomfortable pressure points for all sitters.

Considerations on the subject of contact interaction started with the Hertz solution. Wang and Lakes (2002) report the history of investigations on the contact problems. The classical Hertz theory takes into

\footnotetext{
${ }^{*}$ To whom correspondence should be addressed
} 
account a frictionless interface condition between two 3D elastic elipsoidal deformable bodies in contact. The special case is for contact of an elastic sphere with a halfspace as illustrated in the figure below.

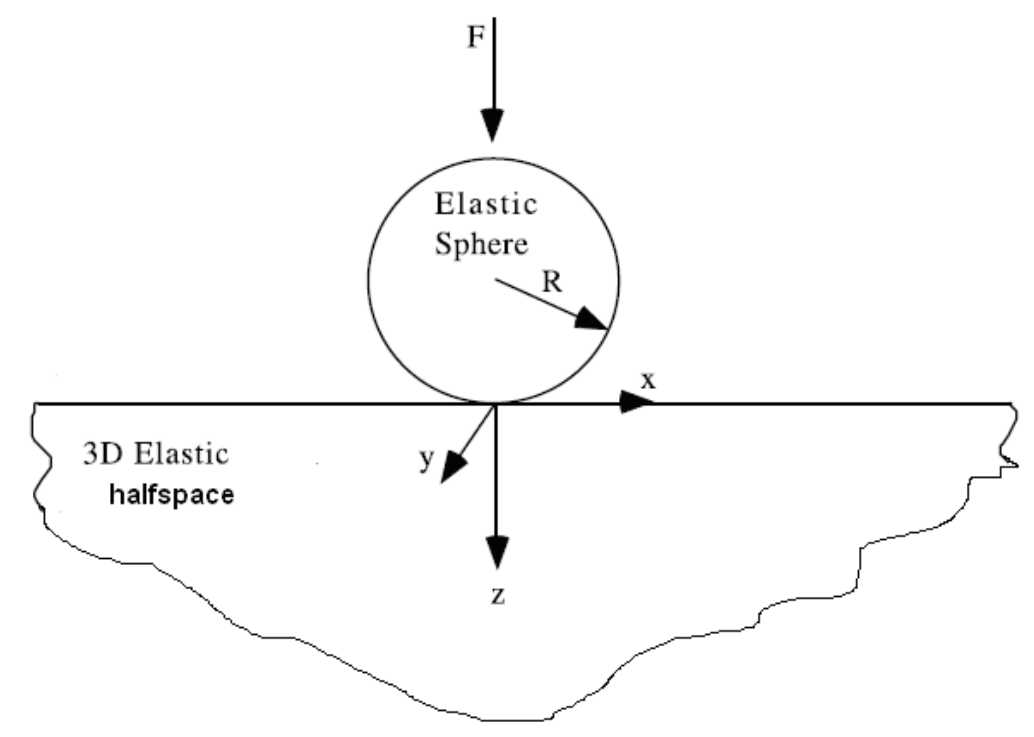

Fig.1. Schematic diagram for Hertz contact model.

The pressure distribution $p(x, y)$ as reported by Wang (2002) is as follows

$$
p(x, y)=p_{\max } \sqrt{1-\frac{x^{2}}{a^{2}}-\frac{y^{2}}{b^{2}}}
$$

where $\quad p_{\max }=\frac{3 F}{2 \pi a b}, \quad a=b=(F D R)^{1 / 3}, \quad D=\frac{3}{4}\left(\frac{1-v_{1}^{2}}{E_{1}}+\frac{1-v_{2}^{2}}{E_{2}}\right)$

$E_{1}, v_{1}$ - material Young's modulus and Poisson's ratio for the halfspace,

$E_{2}, v_{2}$ - material Young's modulus and Poisson's ratio for the sphere.

For the contact of an infinitely rigid sphere $\left(E_{2}=\infty\right)$ with a halfspace the maximum pressure and vertical displacement are as follows

$$
p_{\max }=\frac{3 F}{2 \pi(F D R)^{2 / 3}}, \quad \delta_{\max }=\left(\frac{D^{2} F^{2}}{R}\right)^{1 / 3}
$$

The classical contact problem between a rigid sphere and a half-space elastic layer with finite thickness is described by the Fredholm integral equation. Due to the complexity of the equation, only asymptotic solutions can be obtained. For thick-layer problems, the solutions can be calculated iteratively, but for thin layer ones, the problem can be solved numerically. A closed-form series solution for the contact problem with a finite thickness elastic layer was proposed by Sakamoto et al. (1996). 
Analytical investigations on the contact problems between two homogeneous and isotropic elastic bodies were performed by Wang and Lakes (2002). They also simulated the contact of human buttocks and seat cushions. The cushion materials' Poisson's ratio were allowed to be negative. The human buttocks were modeled as an ideal elastic sphere. The peak contact pressure was reduced by adjusting Poisson's ratio and contour curvature of cushions according to the Hertz theory. An analysis by both the Hertz model and a finite thickness 3D elasticity model showed that using negative Poisson's ratio cushions could further reduce the pressure. Negative Poisson's ratio cushions may be beneficial in the prevention of pressure sores or ulcers in the sick and in reduction of pressure-induced discomfort in seated people. Wang and Lakes investigated the influence of Poisson's ratios, in the full range including negative values on the peak pressure between two contacting elastic bodies and on their deformation.

Generally, a soft cushion helps reduce pressure, but a cushion which is too compliant will bottom out, resulting in increased pressure. The 3D elasticity finite thickness solution incorporates some aspects of bottoming. However a full understanding of bottoming is limited by the complexity of the asymptotic solutions and by the fact that the assumption of linearity excludes the material nonlinearity of the foam and human tissue at high strain. If the cushion's Young's modulus is held constant, the optimal Poisson's ratio is zero. A contoured cushion reduces peak pressure. Combining the concepts of contoured shape and negative Poisson's ratio materials offers superior cushion performance. An analysis which incorporates more of the complexities of the problem such as material nonlinearity should incorporate the finite element method treatment.

\section{Formulation of the contact problem}

For the unilateral static contact problem of a linear elastic body with a stiff body the following system of equations must be fulfilled (Kikuchi and Oden, 1988)

$$
\sigma_{i j, j}+f_{i}=0, \quad \sigma_{i j}=S_{i j k l} \varepsilon_{k l}, \quad \varepsilon_{i j}=\frac{1}{2}\left(u_{i, j}+u_{j, i}\right) \quad \text { in } \quad \Omega,
$$

completed with boundary conditions

$$
u_{i}=\hat{u}_{i} \quad \text { on } \quad \Gamma_{D}, \quad \sigma_{i j} \cdot n_{j}=t_{i} \quad \text { on } \quad \Gamma_{F},
$$

contact conditions on $\Gamma_{C}$

$$
\sigma_{n} \cdot\left(u_{n}-g\right)=0, \quad \sigma_{n} \leq 0, \quad u_{n}-g \leq 0,
$$

and friction conditions on $\Gamma_{C}$

$$
\left|\boldsymbol{\sigma}_{T}\right|<\mu\left|\sigma_{n}\right| \Rightarrow \Delta \boldsymbol{u}_{T}=0, \quad\left|\boldsymbol{\sigma}_{T}\right|=\mu\left|\sigma_{n}\right| \Rightarrow \exists \lambda>0 ; \quad \Delta \boldsymbol{u}_{T}=-\lambda \boldsymbol{\sigma}_{T}
$$

where: $\sigma_{i j}$ - Cauchy stress tensor, $\varepsilon_{i j}$-small strain tensor, $S_{i j k l}$ - elastic stiffness matrix, $u_{i}$ - displacement vector, $f_{i}$-body forces, $\hat{u}_{i}$ - prescribed displacements on $\Gamma_{D}, t_{i}$ - forces acting on $\Gamma_{F}, n_{i}$-unit normal vector, $\Gamma_{D} \cup \Gamma_{F} \cup \Gamma_{C}$ - boundary of the domain $\Omega, g$ - initial gap, $\sigma_{n}=\sigma_{i j} n_{i} n_{j}$ - contact pressure, $u_{n}=\boldsymbol{u} \circ \boldsymbol{n}$ displacement normal to the boundary, $\sigma_{T i}=\sigma_{i j} \cdot n_{j}-\sigma_{n} \cdot n_{i}-$ tangential contact force, and $\Delta \boldsymbol{u}_{T}=\Delta\left(\boldsymbol{u}-u_{n} \cdot \boldsymbol{n}\right)$-increment of tangential displacement.

To solve the boundary value problem formulated above (nonlinear due to conditions (2.3) and (2.4)) the FEM approach is used. 


\section{The main idea of seat design}

Negative Poisson's ratio materials are called auxetics. They are classified as smart materials having a significant technological potential. An example of auxetics are some cellular materials. Cellulars with various microstructures constitute a special kind of elastic media. The auxecity in the selected directions and required magnitude of Poisson's ratio may be tailored according to the designer demands by a proper choice of microstructural geometrical parameters (Janus-Michalska, 2009). The theoretical problem of material design for anisotropic elastic cellular bodies with respect to contact problems is presented by Jasińska and Janus-Michalska $(2008 ; 2010)$. Special attention is paid to materials having reentrant structure, which yield negative Poisson's ratio. These properties and the orientation of material symmetry axes with respect to load direction significantly influence contact stress distribution and may play an important role in reducing the peak contact pressure.

This work discusses the application of auxetic skeleton structures in seats. The seat skeleton is composed of 'springs', which are based on auxetic frame structures designed similarly to the microstructure of auxetic cellular materials as described by Jasińska and Janus-Michalska (2012).

Seat design is an example of application of the features mentioned above. A new design can be tested by computer simulations using models of the human body and the seat.

Overall seat comfort is also influenced by seat characteristics (e.g., seat stiffness) as reported by Ebe and Griffin (2000). Progressive characteristics of an elastic medium give an advantegous distribution of pressure. Progressive means that the seat is compliant for a smaller load and becomes stiffer for incresasing loads. The skeleton structure is designed to work in two consecutive stages. At first, as an elastic response at small deformations, the network deforms fairly uniformly. Then, under increasing loading, the local elastic collapse occurs, leading to an increased bending in the network. As the structural configuration evolves, new weak points are created and high degrees of bending propagate throughout the structure which results in large deflections. Finally, the network collapses onto itself and self-contact between frame elements results in the stiffening of the structure.

A three-dimensional real contact and stiffness problem of small size auxetic springs constituting a part of an elastic seat cushion is considered. The analysis is performed using FEM. Computer simulations of the seat structure under a typical static loading exerted by a human body were performed by means of ABAQUS FEA (calculations were performed at ACK CYFRONET AGH). Extensive numerical simulations were carried out using nonlinear analysis to calculate the stresses and deflections.

The finite element model of a seat, for both seats could provide an insight into changes in contact interaction between the human and the seat due to variations of geometric and material parameters.

\section{Seat structural elements}

Two types of seats as illustated in Fig.1 are considered. In each case a half of the seat structure with appropriate load and symmetry boundary conditions is analysed. A foam seat is composed of various foam elements: seat filling, bodno and upper seat layer. In an auxetic seat the seat filling is replaced by an auxetic skeleton whereas the remaining parts remain unchanged.

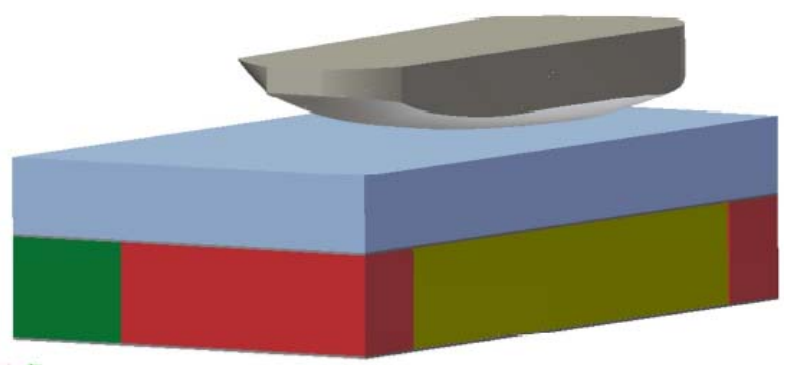

a. 


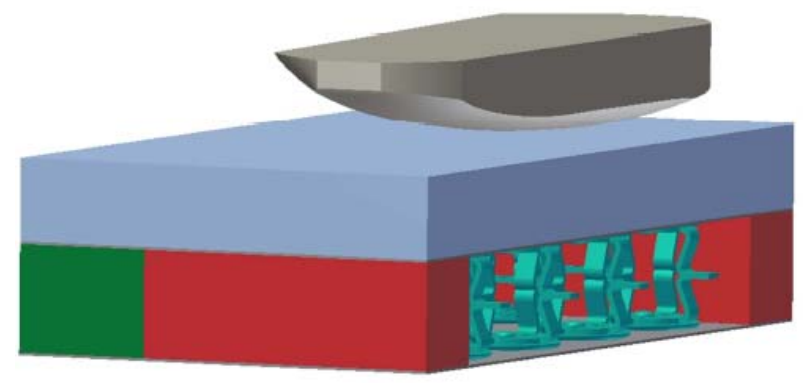

b.

Fig.1. Structures of half seat with intender. a. foam seat b. seat with auxetic spring skeleton.

The spring skeleton consists of silicon springs of the shape illustrated in the figure below. Special rubber discs are applied to the bottom and top surfaces of the spring skeleton elements to ensure a proper interaction with the aligning felt layer (Fig.2).

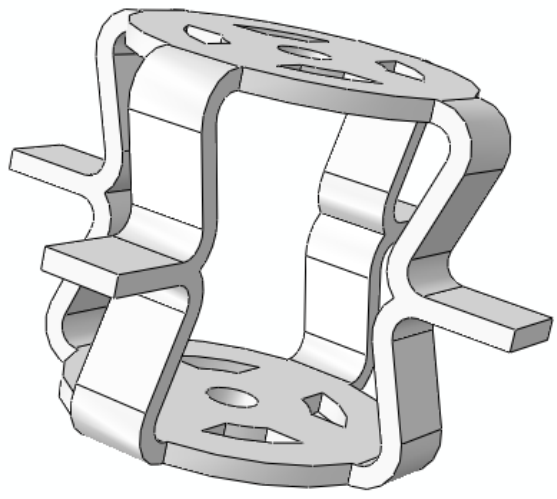

$\mathrm{a}$

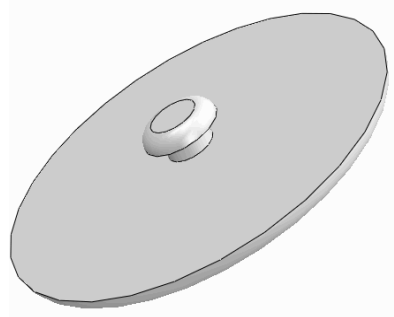

b

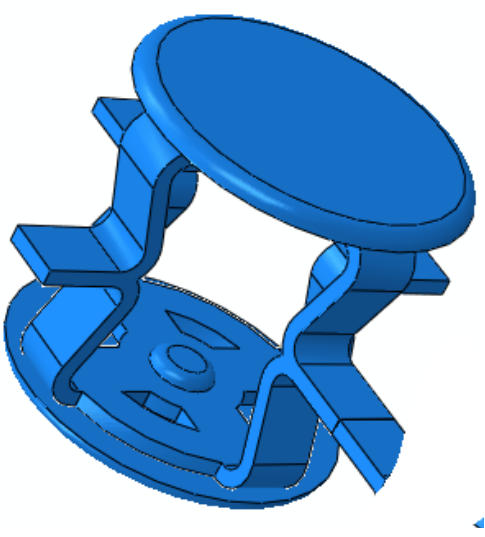

$\mathrm{c}$

Fig.2. Skeleton elements, a. auxetic silicon spring, b. rubber disc, c. spring with two discs.

\section{Material data}

The specification of materials for structural elements is given in Tab.1.

Table.1. Mechanical properties of seat structural elements materials.

\begin{tabular}{|l|l|l|}
\hline seat part & material & elastic properties \\
\hline upper seat layer & foam T3037 & nonlinear as given in Fig.3a \\
\hline seat filling (seat a) & foam T3037 & nonlinear as given in Fig.3a \\
\hline bodno & foam T3546 & nonlinear as given in Fig.3b \\
\hline auxetic spring (seat b) & silicon 75 Shore'a & $\begin{array}{l}\text { Mooney-Rivlin hyperelastic model } \\
E=9.32[\mathrm{MPa}], C 10=1.05, C 01=0.3, K=7.77[\mathrm{MPa}]\end{array}$ \\
\hline disc(seat b) & Shore rubber 95 & $E=4000[\mathrm{MPa}], v=0.3$ \\
\hline aligning layer & felt & $E=2.58[\mathrm{kPa}], v=0.3$ \\
\hline
\end{tabular}


a)

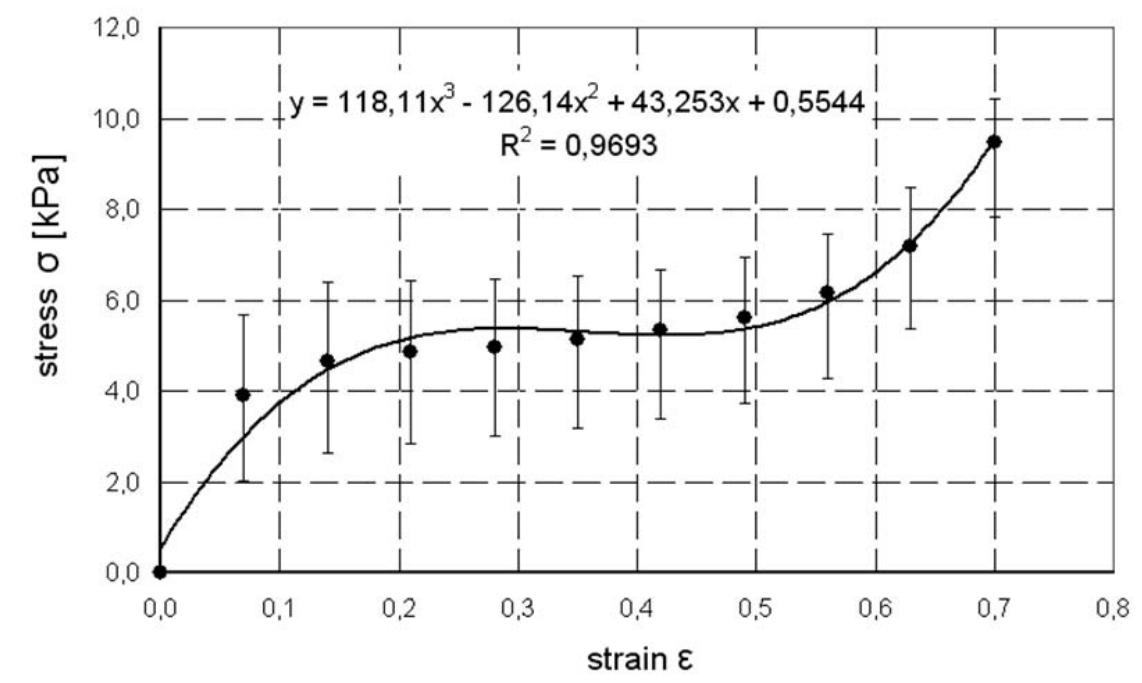

b)

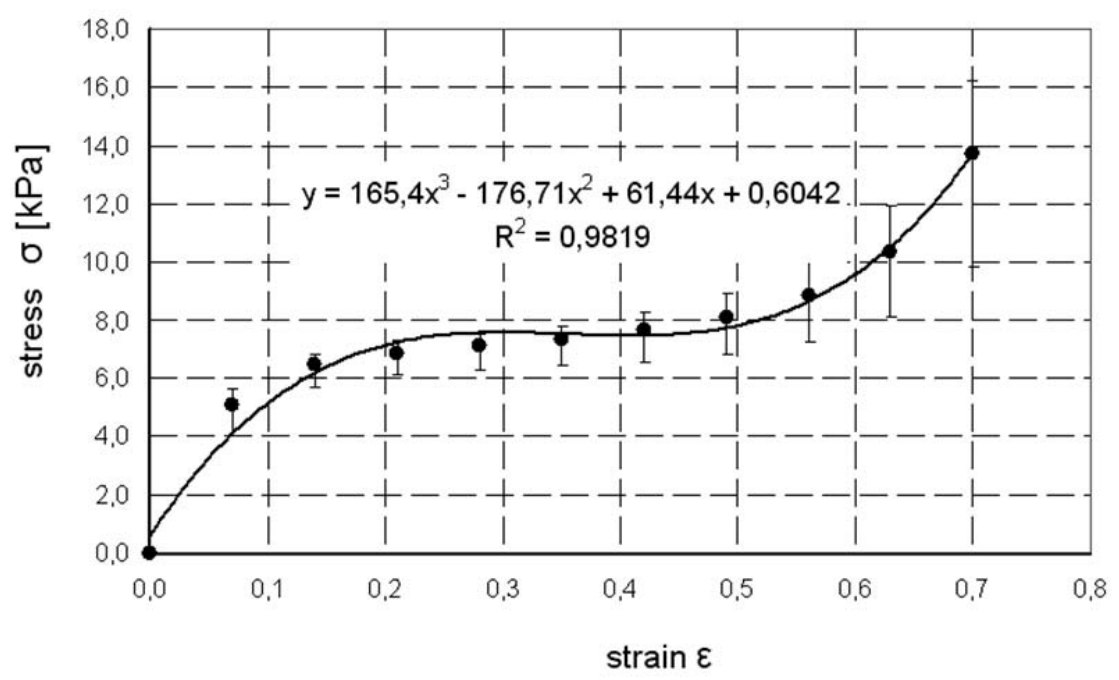

Fig.3. Nonlinear foam characteristics, a. foam T 3037, b. foam T 3546.

Seat dimensions: height $138 \mathrm{~mm}$, width $640 \mathrm{~mm}$, depth $580 \mathrm{~mm}$.

\section{Seat design requirements}

The following two states are considered: ultimate limit stress state and serviceability limit state. The first state reads that the reduced von Mises stress at each point of the structure fulfills the condition: $\sigma^{M} \leq f_{d}$ where: $f_{d}$ denotes limit stress value. The serviceability limit state concerns vertical deflection, which should not exceed $50 \%$ of seat height. Comfort condition means that contact stresses are limited by the value of $4.265 \mathrm{kPa}$ - the limit value of pressure. 


\section{Numerical analysis - scope of the study}

In this paper, we perform a numerical analysis to determine the nonlinear load displacement path for static loading (seat stiffness characteristics). We also present stress maps. The strength of given structures for static loading is checked by reduced Mises stress maps. Calculations are performed for utility load represented by an indenter of the shape of a human thigh and buttock.

\subsection{Finite element mesh}

\section{a. Foam seat}

56000 8-node linear brick elements, (reduced integration, enhanced hourglass control) modeling aligning foam and bodno foam,

2400 3-node triangular facet rigid elements for model of indenter's surface.

\section{b. Auxetic spring seat}

preliminary test on single spring -55600 4-node linear tetrahedron elements

Seat with auxetic skeleton - discretization of seat elements spring skeleton - 9400010 -node quadratic tetrahedron elements bodno and aligning foam- 822008 -node linear brick elements felt - 5100 4-node quadrilateral membrane elements intender- 24003 -node triangular facet rigid elements.

\subsection{Type of analysis}

A geometric nonlinear analysis (large displacements and contact between seat elements) is performed. Material nonlinearity is also involved due to material nonlinearity of hyperelastic foams.

\subsection{Boundary conditions and contact conditions}

Half of the seat structure with load due to symmetry is analysed. Static loading is performed by kinematic exertion - intender displacement. The intender is modeled as a rigid body, contact with the seat is frictionless, bodno parts are stuck together, bodno and felt are also stuck together.

Seat a. frictionless contact between foam and bodno.

Seat $b$. additional conditions: spring and discs are stuck together, parts of bodno and felt are sticked together, friction coefficient disc-felt 0.1 or without friction, self contact of spring is frictionless.

\subsection{Contact stress distribution and seat deformation}

\section{Foam seat}

A nonlinear incremental analysis is performed for seat deflection egual to $50 \%$ of its height (with corresponding loading $300 \mathrm{~N}$ ). Maps of contact stress distribution are given in Fig.4. For increasing loading the maximum contact stress also increases. The maximum stress value is higher than $4.265 \mathrm{kPa}$ - the limit value of comfort condition. Figure 5 illustrates deformation in subsequent loading stages. 

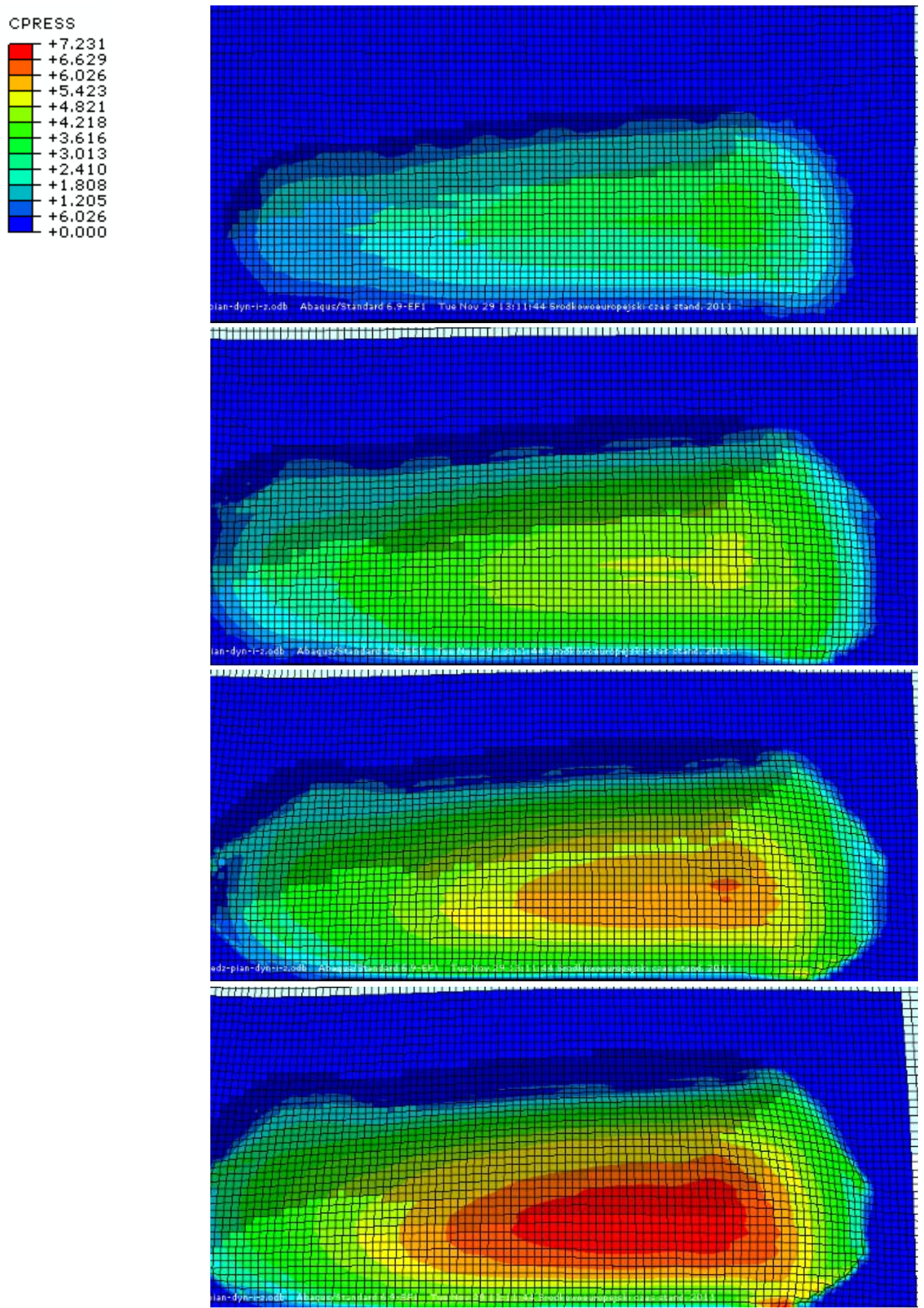

Fig.4. Contact stress distributions $[\mathrm{kPa}]$ in subsequent equal load increments to maximal loading $(300 \mathrm{~N})$. 

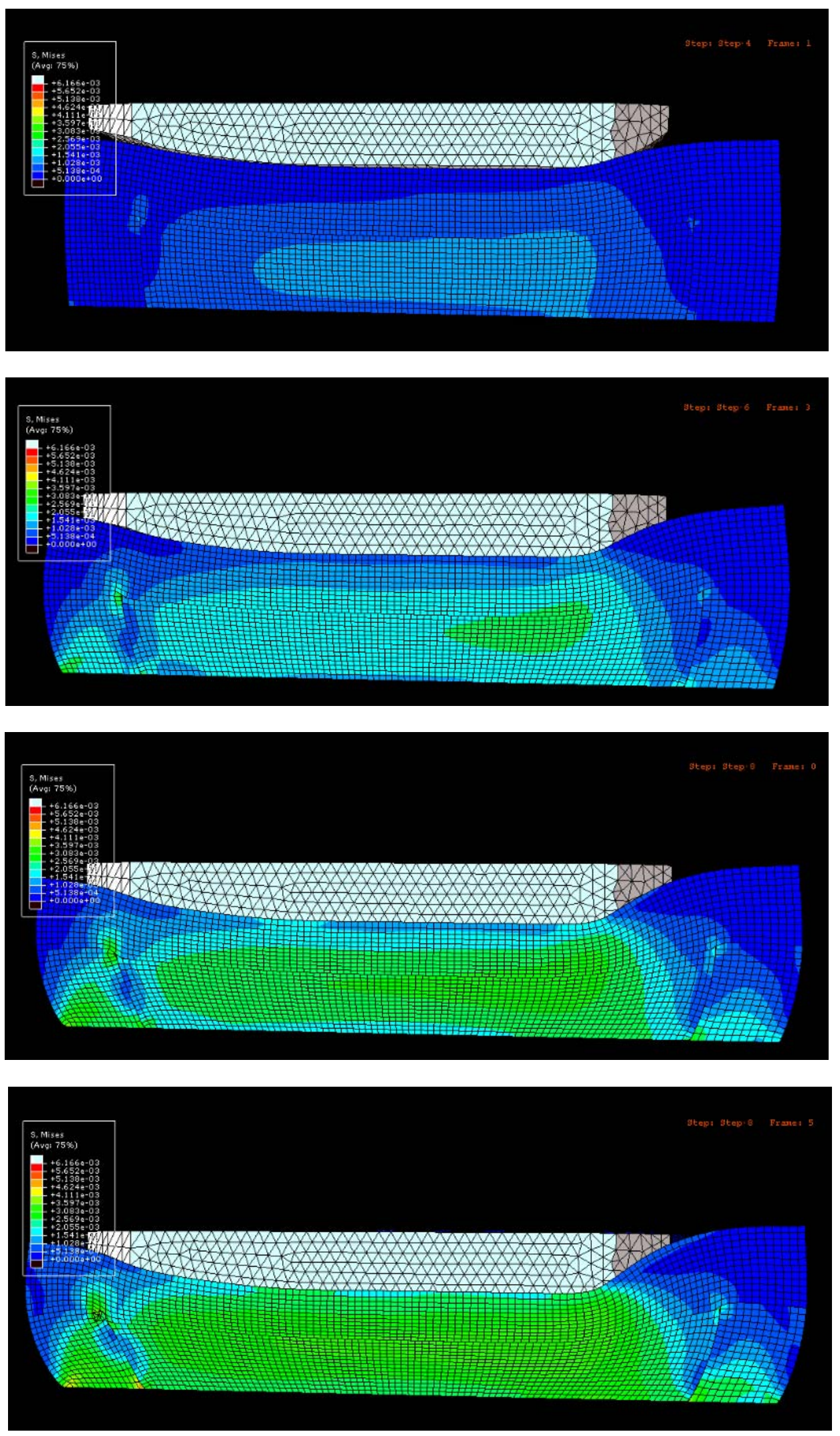

Fig.5. Deformation in subsequent equal load increments to maximal loading (300N). 


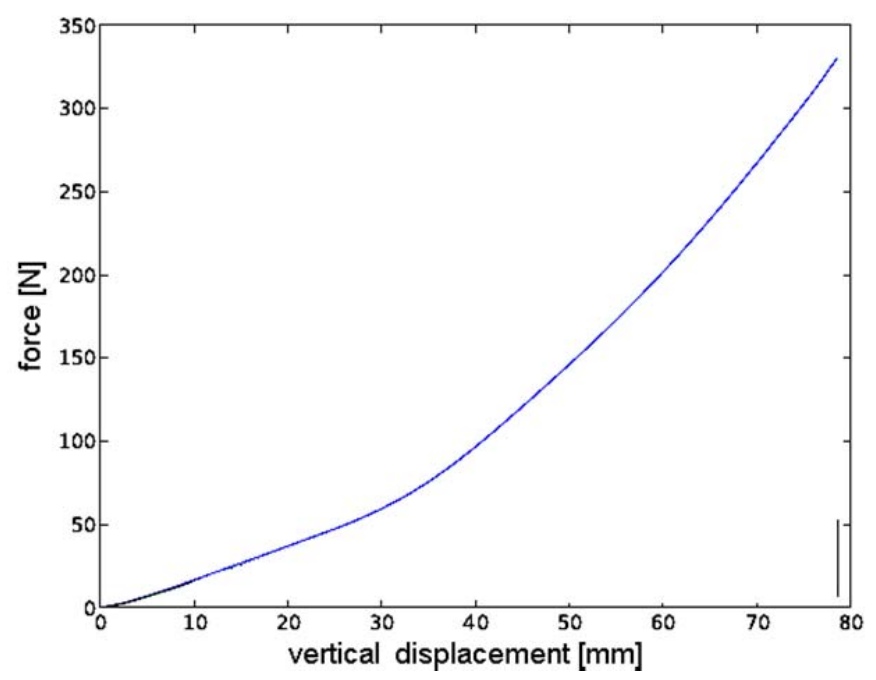

Fig.6. Load-displacement path.

Figure 6 shows the nonlinear load- displacement path.

\section{Auxetic seat}

As a preliminary task we analyze the deformation and load bearing capacity of a single spring compressed by a stiff plane surface. The friction coefficient between discs and the surface $\mu$ equals 0.2 .

\section{S, Mises}

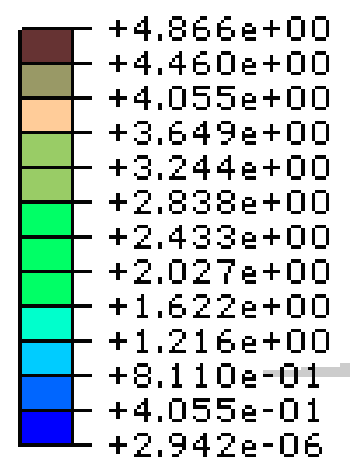

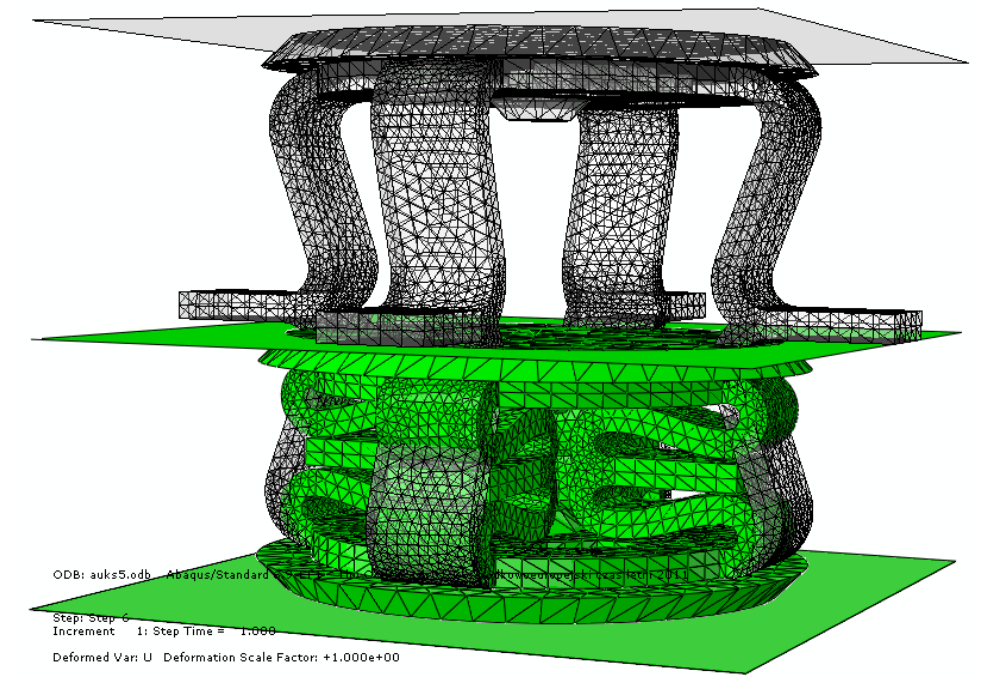

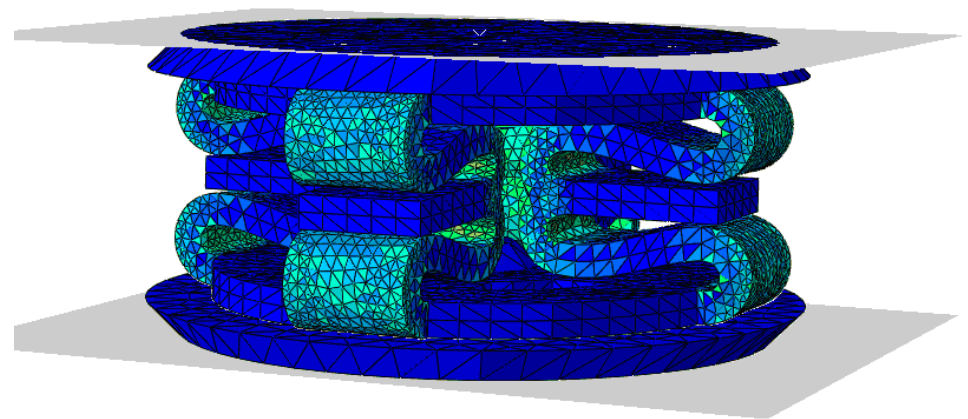

Fig.7. Deformation and stress distribution [MPa]. 
The average pressure simulated by the stiff surface loaded by a typical weight of a human body is $0.0210^{6}$ $N / m^{2}(20 \mathrm{kPa})$ and maximum pressure is $0.07210^{6} \mathrm{~N} / \mathrm{m}^{2}(72 \mathrm{kPa})$. It is recommended for the average pressure to deliver a vertical displacement of $20 \%-30 \%$ of spring height, and for the maximum pressure the vertical displacement should not exceed $40 \%$ of spring height.

The maximum deformation and corresponding stress distribution are given in Fig.7 to check if a material fulfills the first design condition $\left(f_{d}=20 M P a\right)$.

\section{Seat with auxetic spring skeleton}

We consider two cases with respect to friction coefficient.

Case 1. Friction coefficient between the rubber discs and felt $\mu=0.1$

Distributions of contact pressure stress $[\mathrm{kPa}]$ and deformation in subsequent equal load increments (for maximal seat deflection equal to $50 \%$ of its height) are illustrated in Fig.8. It is clearly visible that redistribution of contact pressure (after fourth increment) gives lower pressure maximum.
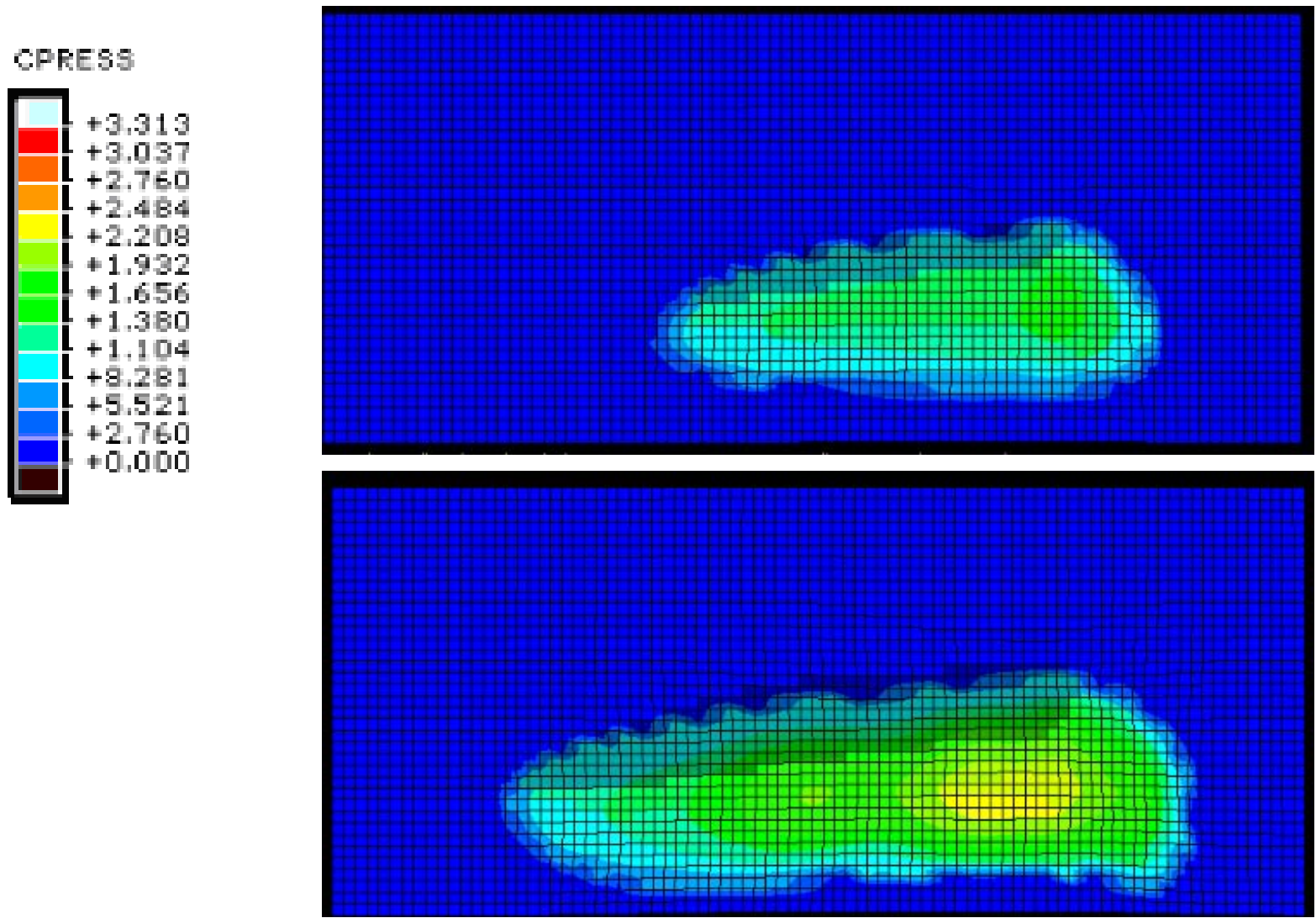

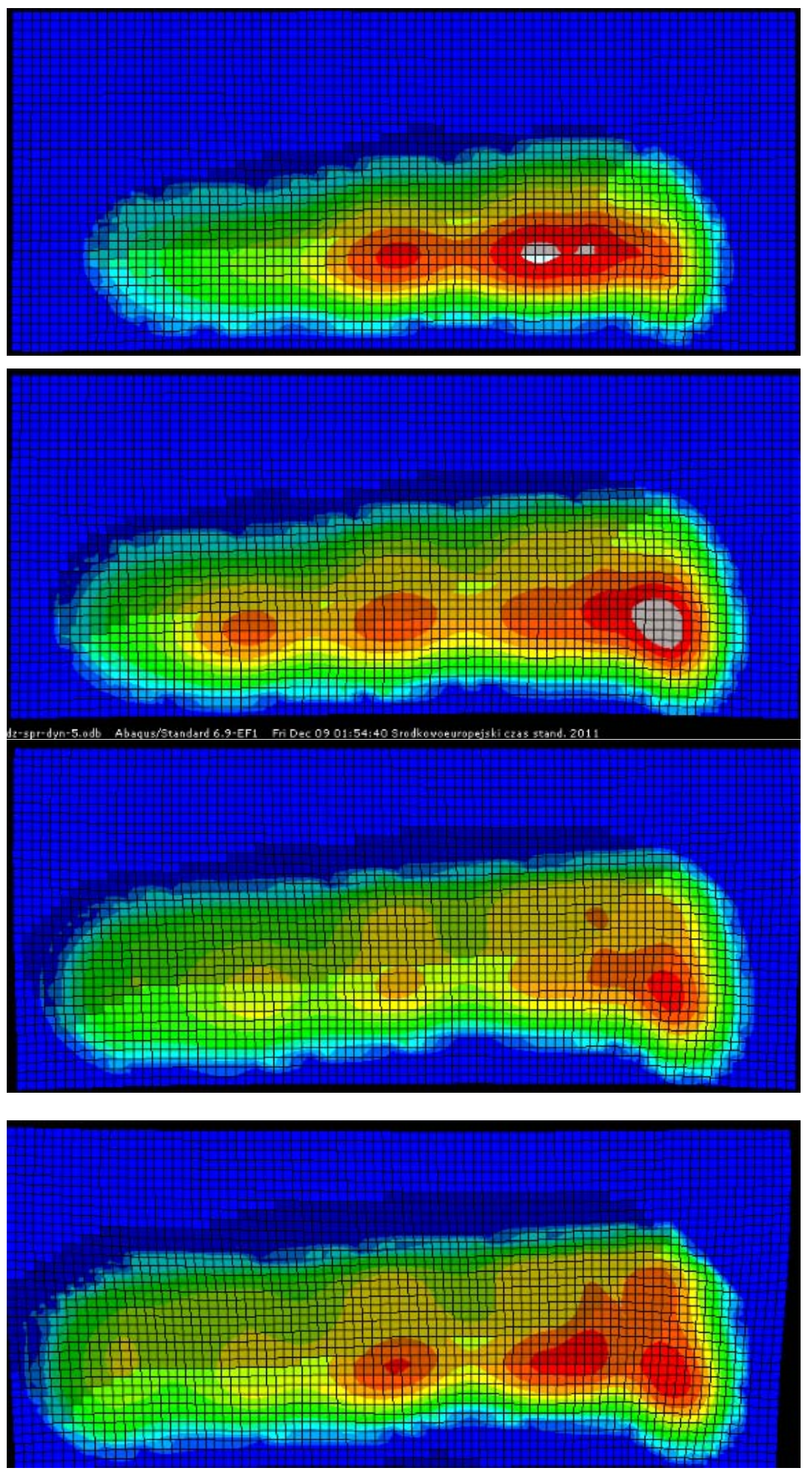

Fig.8. Distribution of contact pressure stress $[\mathrm{kPa}]$ in subsequent equal load increments. 

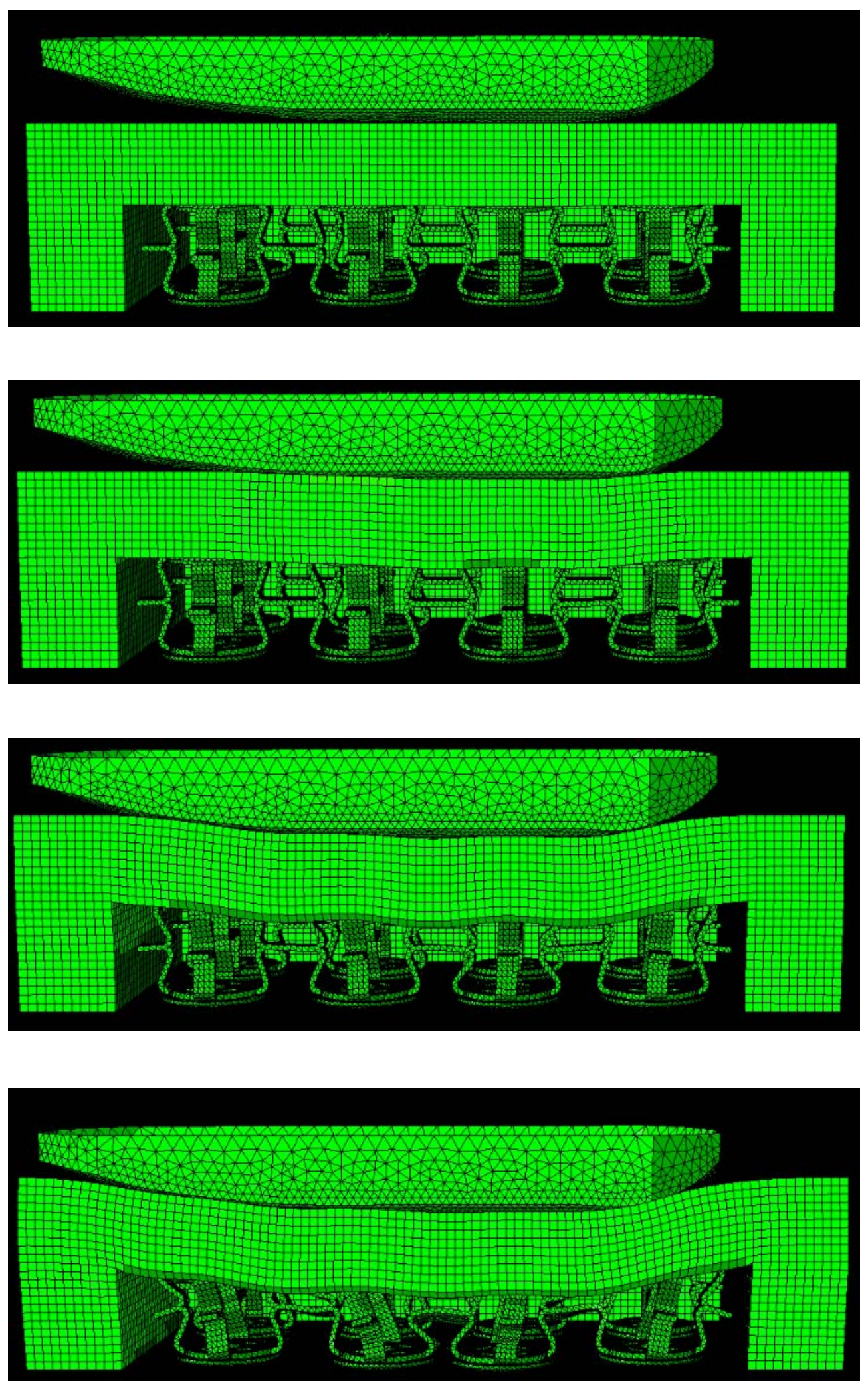

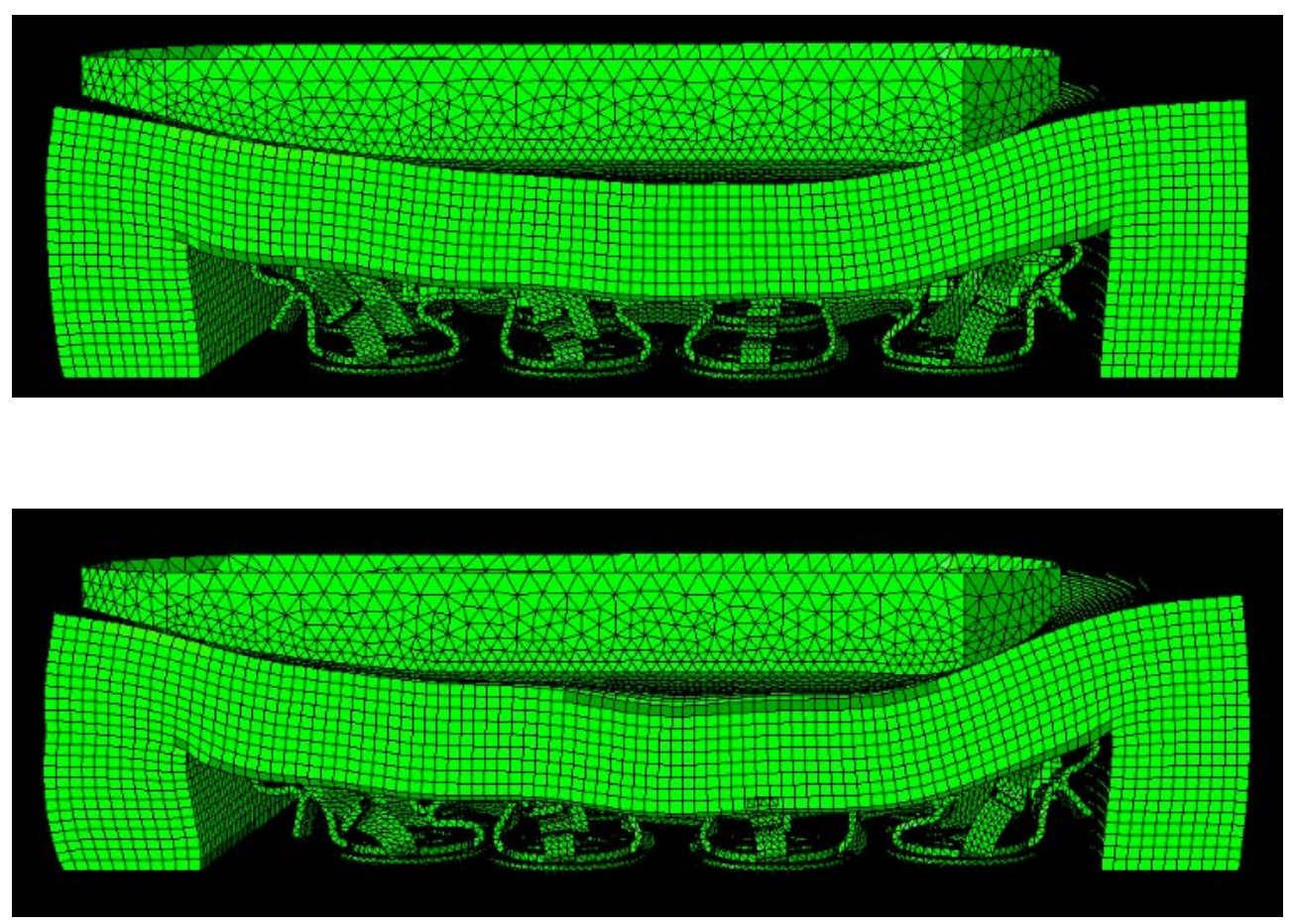

Fig.9. Seat deformation in subsequent equal load increments.

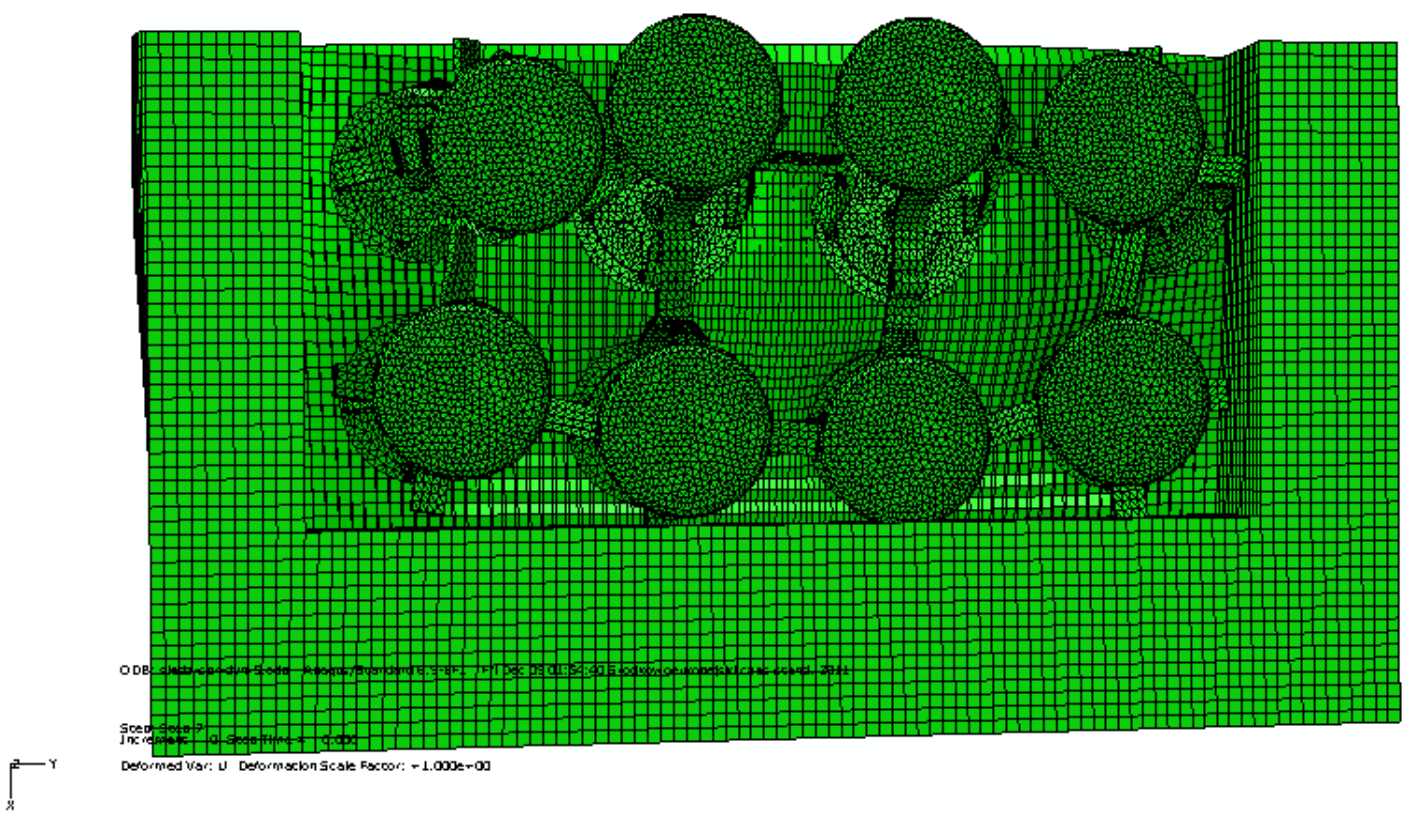

Fig.10. Horizontal displacements of springs (for maximal loading).

Seat characteristics is illustrated below (in Fig.11). 


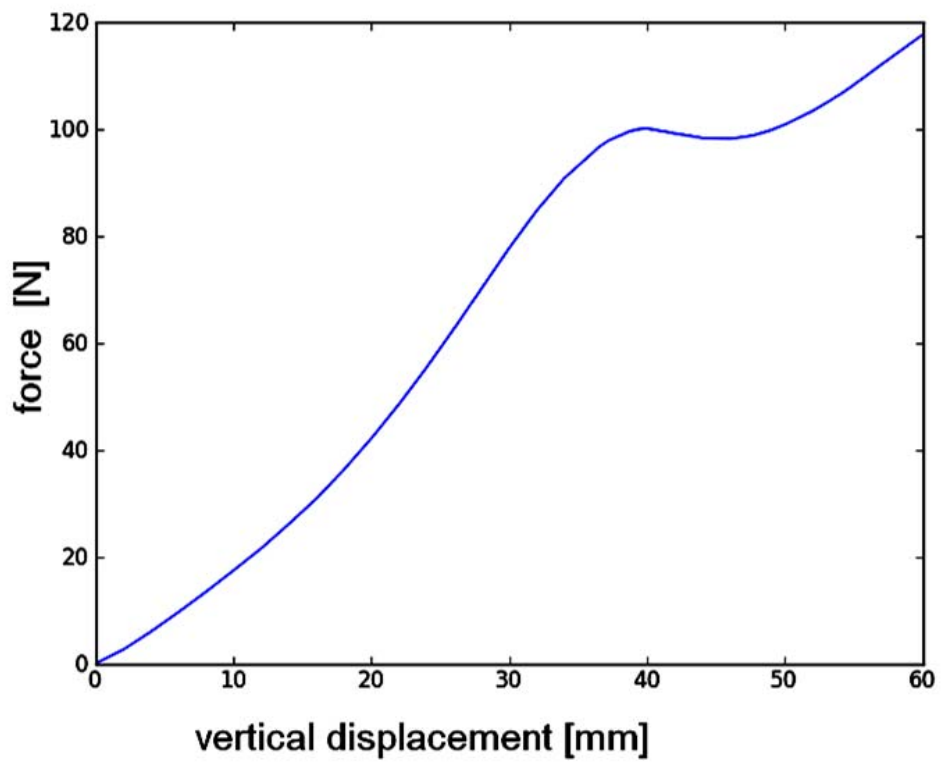

Fig.11. Load-vertical displacement path.

Case 2. Frictionless contact between the rubber disc and felt $(\mu=0)$.

For comparison with the previous example distributions of contact pressure stress [kPa] and deformation are illustrated in figure below. An advantageous redistribution of contact pressure is also visible.

CPRESS
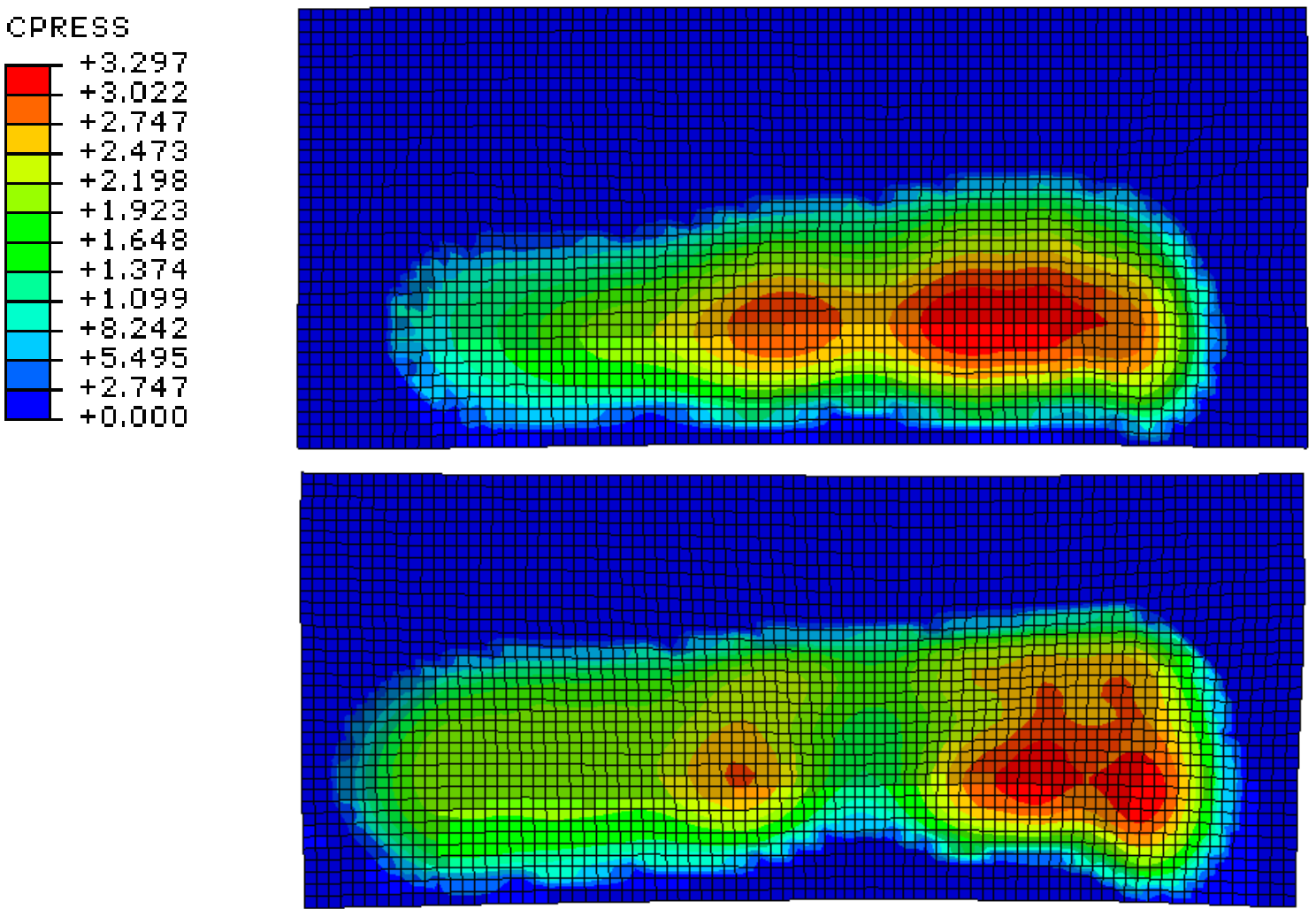

Fig.12. Distribution of contact pressure $[\mathrm{kPa}]$ for deflections of $30 \mathrm{~mm}$ and $50 \mathrm{~mm}$. 


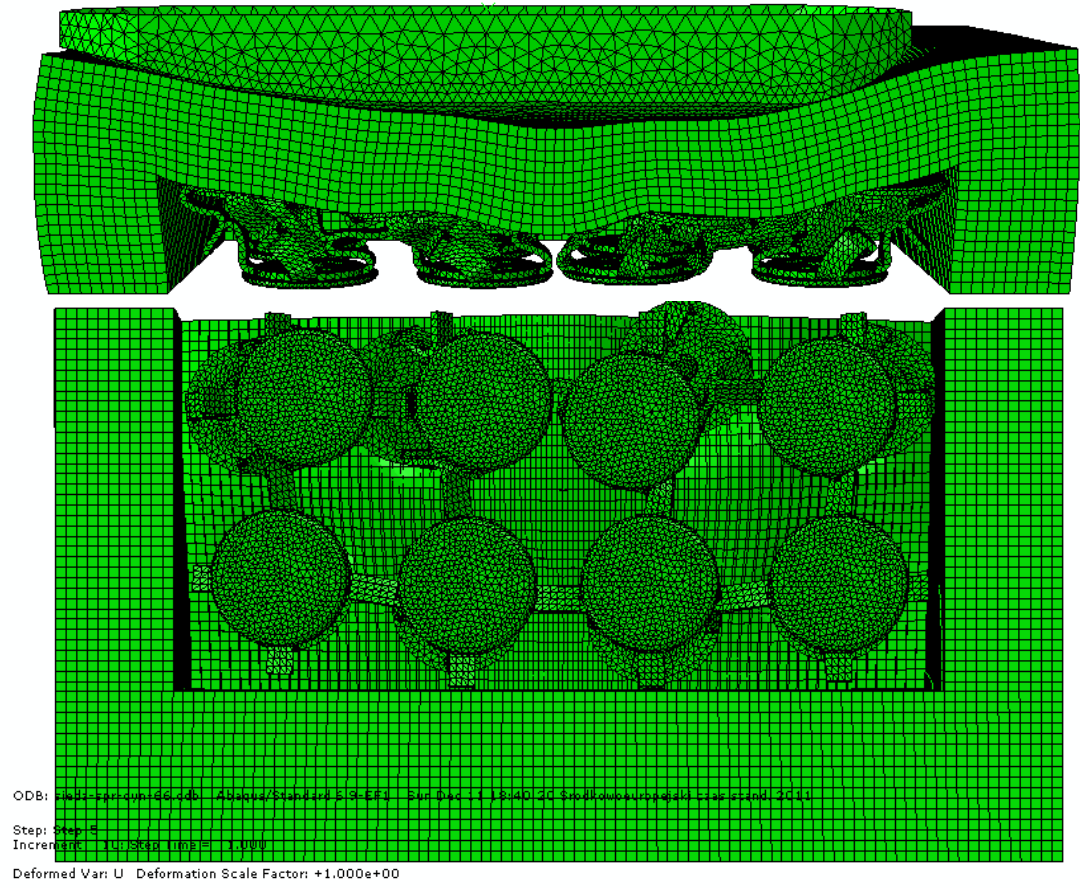

Fig.13. Deformation and horizontal displacements of springs (for maximal deflection).

Seat characteristics is illustrated below.

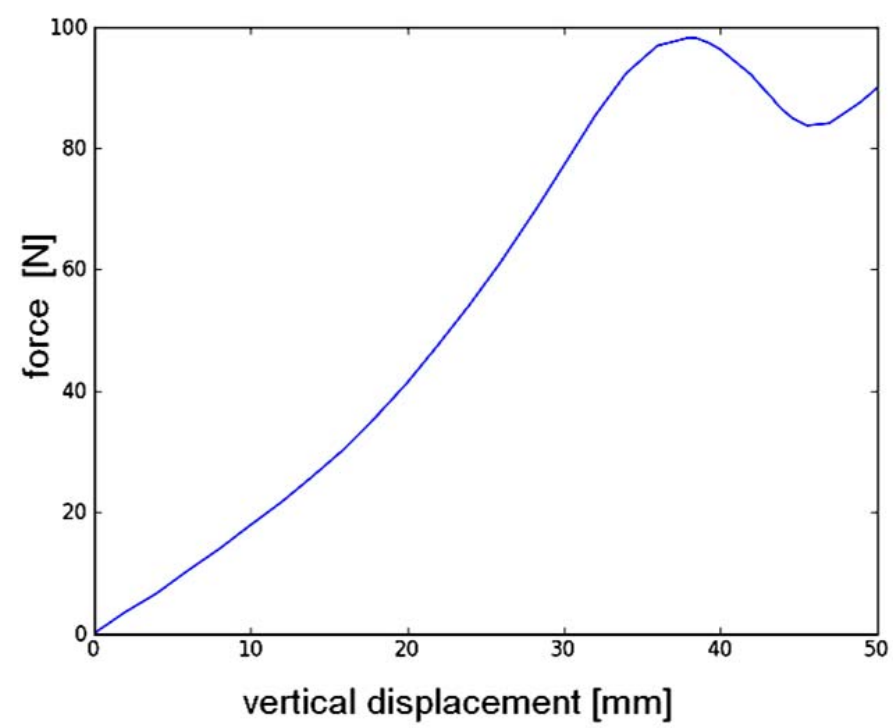

Fig.14. Nonlinear load-displacement path.

\section{Conclusions}

The finite element models of two types of seats are developed for prediction of structure deformation modes, load bearing capacity, and seat pressure distributions at the contact interface between a human body and a seat. The numerical results confirm the suggestion that a negative Poisson's ratio seat skeleton reduces the stress concentrations and gives an advantageous stress redistribution, which fulfills comfort condition. The analysis allows the geometrical nonlinearity due to contact and large displacements and also material 
nonlinearity of seat components. A short parameter study shows that seat pressure distributions are sensitive to variations in the seat properties. The structures are not adjusted to full bearing capacity. Changes of geometrical and material parameters can optimize the seat structure. The insight gained from the present analysis may be of use in designing new seats, and also provide guidelines for future analysis which can incorporate more complexities of the problem.

\section{Nomenclature}

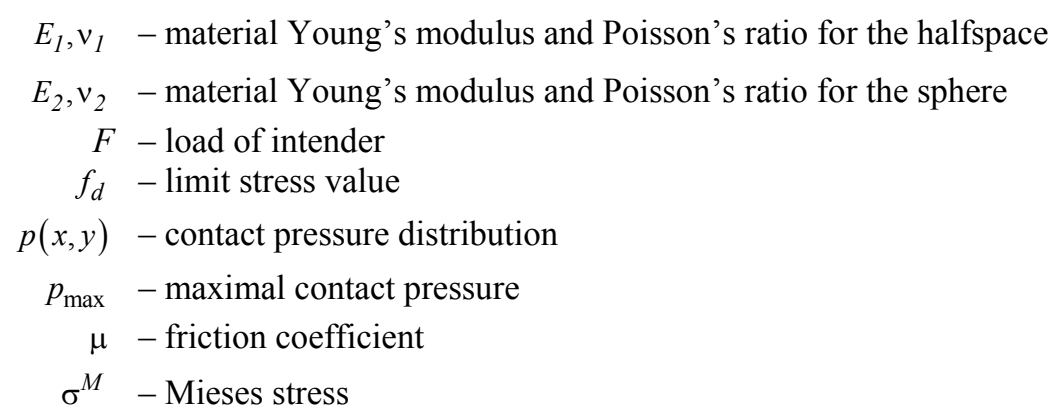

\section{References}

Cassar T. and Gross C.M. (1995): Evaluation of an intelligent seat system. - Applied Ergonomics, vol.26, pp.109-116.

Ebe K. and Griffin M.J. (2001): Factors effecting static and seat cushion comfort. - Ergonomics, vol.41, No.10, pp.901-992.

Ebe K. (1998): Predicting overall seat discomfort from static and dynamic characteristics of seats. - PhD thesis, Institute of Sound and Vibration Research, Faculty of Engineering and Applied Science, University of Southampton, UK.

Janus-Michalska M. (2009): Micromechanical model of auxetic cellular materials. - Journal of Theoretical and Applied Mechanics (JTAM), 4, vol.47 pp.737-750.

Janus-Michalska M. and Jasinska D. (2010): Contact problem for a class of anisotropic elastic cellular bodies with nonpositive Poisson's ratio. - Technical Transactions, 4-B/2010/issue 19, year 107.

Jasińska D. and Janus-Michalska M. (2008): Material design of anisotropic elastic cellular bodies with respect to contact problem - Engineering Transactions, vol.56, No.3, pp.201-225.

Jasińska D., Janus-Michalska M. and Smardzewski J. (2012): A study on the design of auxetic structure of seat skeleton. - In Review for Mechanics and Control.

Johnson K.L. (1985): Contact Mechanics. - Cambridge, London: Cambridge University Press.

Keer L.M. (1964): The contact stress problem for an elastic sphere indenting an elastic layer. - Journal of Applied Mechanics, pp.143-145.

Kikuchi N. and Oden J.T. (1988): Contact Problems in Elasticity: A study of Variational Ineqalitiees and Finite Element Methods. - SIAM Philadelphia.

Lowe A. and Lakes R.S. (2000): Negative Poisson's ratio foam as seat cushion material. - Cellular Polym., vol.19, No.3, pp.157-167.

Moes C. (1989): The development of a pressure distribution measuring device for various person-product contact areas. - Proceedings of the Ergonomics Society's 1989 Annual Conference”, Contemporary Ergonomics, pp.349354, Reading, UK.

Moes C. (1999): Proceeding of the ISATA Automotive Mechatronics Computer Support for Pressure Distribution Controlled Shape Design Design \& Engineering, Vienna, pp.483-492.

Park S.J. and Kim C.B. (1997): The evaluation of seating comfort by objective measures. - SAE Conference. 
Park S.J. and Lee Y.S., Nahm Y.E., Lee J.W., Kim J.S. (1998): Seating physical characteristics and subjective comfort: design considerations, SAE Conference, SAE no 980653.

Sakamoto M., Li G., Hara T. and Chao Y.S. (1996): A new method for theoretical analysis of static indentation test. - J. Biomech., vol.29, No.5, pp.679-685.

Staarink H. (1995): Sitting Posture, Comfort and Pressure. - PhD Thesis, Delft University of Technology, Delft, the Netherlands.

Swearingen J.J., Wheelwright C.D. and Garner J.D. (1962): An analysis of sitting areas and pressure of man (Report 62-1), Oklahoma City, OK: Civil Aero Medical Research Institute.

Verver M.M., van Hoof J., Oomens C.W.J., Wismans J. and Baaijens F.P.T. (2004): A finite element model of the human buttocks for prediction of seat pressure distributions. - Computer Methods In Biomechanics and Biomedical Engineering, vol.7, No.4, pp.193-203.

Wang Y. and Lakes R. (2002): Analytical parametric analysis of the contact problem of human buttocks and negative Poisson's ratio foam cushions. - International Journal of Solids and Structures, vol.39, pp.4825-838.

Zhao L.Q., Xia Q.S. and Wu X.T. (1994): Study of sitting comfort of automotive seats. - SAE Conference 1994, SAE No 945243.

Received: April 10, 2012

Revised: April 12, 2012 